\title{
Asymptotic properties of least squares estimation for a new fuzzy autoregressive model
}

\author{
Zhi-Wen Zhao $^{1 *}$ and Cui-Xin Peng ${ }^{2}$
}

\section{"Correspondence:}

zhaozhiwen@126.com

${ }^{1}$ College of Mathematics, Jilin

Normal University, Siping, 136000,

P.R. China

Full list of author information is

available at the end of the article

\begin{abstract}
In this paper, we extend the standard autoregressive model to the case where the explanatory and response variables are random fuzzy variables. The fuzzy

least-squares estimators (FLSE) of the model parameters are derived and their asymptotic properties are established. A simulation is conducted to evaluate our method, and it is found that the proposed method provides a better performance.
\end{abstract}

AMS Subject Classification: 94D05; 62F12

Keywords: fuzzy random variables; fuzzy numbers; fuzzy least squares estimation; fuzzy autoregressive model; fuzzy set

\section{Introduction}

The time series forecasting investigates the relations on the sequential set of past data measured over time to forecast the future values. The area has been widely studied, and traditional forecasting is frequently conducted by statistical tools like regression analysis, moving average, integrated moving average, and autoregressive integrated moving average.

However, the deficiencies of traditional forecasting methods are that they cannot deal with forecasting problems in which historical data are linguistic values. In order to overcome the drawback of the traditional forecasting methods, in [1], Song and Chissom proposed the concepts of fuzzy time series to investigate the forecasting problem in which historical data are linguistic values. In [2] and [3], they proposed two fuzzy time series models to study the forecasting problems of enrollments of the University of Alabama. Some researchers such as $[4,5]$, and [6] have also proposed new fuzzy time series models to improve Song's model. These fuzzy time series models have been applied to various practical questions such as temperature by Chen \& Hwang [7], the stock index by Huarng [8], Huarng \& $\mathrm{Yu}[9,10]$, and $\mathrm{Yu}[11,12]$ etc.

In particular, Ozawa [13] proposed a fuzzy auto-regressive (AR) model to forecast the data of living expenditure of workers' household in Japan, where the identification and the estimation of its model and the model parameters are optimized by the linear programming problem under some conditions. Moreover, Niimura [14] presented a fuzzy autoregressive model to estimate uncertain electricity market prices in deregulated industry environment, and parameters are also obtained by solving linear programming problems.

(c) 2013 Zhao and Peng; licensee Springer. This is an Open Access article distributed under the terms of the Creative Commons Attribution License (http://creativecommons.org/licenses/by/2.0), which permits unrestricted use, distribution, and reproduction in any medium, provided the original work is properly cited. 
Furthermore, the uncertainty of observations or the uncertainty of the model have been treated as having a stochastic error, and we generally analyze those data or system with the stochastic approach. In fact, Kim et al. [15] pointed out that two different kinds of uncertainty, vagueness and randomness, coexist within a model. Randomness resulting from measurement errors and fuzziness resulting from system fuzziness are two different kinds of uncertainty. Therefore, he extended the standard linear regression model to include specific cases where the observations are vague or even linguistic. In this paper, we further discover the analogous results for autoregressive models in time series analysis. Consider the following new fuzzy autoregressive model:

$$
X_{t}=\alpha X_{t-1} \oplus \Phi_{t}, \quad t=1,2, \ldots
$$

where $X_{0}$ is a constant triangular fuzzy number, $X_{t}$ are random fuzzy variables which are expressed by $\left(x_{t}, \xi_{t}^{l}, \xi_{t}^{r}\right)_{\triangle}$ with crisp random variables $x_{t}, \xi_{t}^{l}, \xi_{t}^{r}, \Phi_{t}=\left(\varepsilon_{t}, \theta_{t}^{l}, \theta_{t}^{r}\right)_{\triangle}$ are the fuzzy random errors, and $|\alpha|<1$ is unknown regression crisp parameter to be estimated on the basis of fuzzy observations $X_{t}$. Obviously, our model is different from the models that are discussed above. Moreover, this paper is devoted to the parameter estimation of the model and sets out the asymptotic properties of the estimation.

The rest of this paper is organized as follows. Section 2 briefly introduces the literature related to fuzzy sets, fuzzy numbers, and triangular fuzzy numbers (TFN), fuzzy time series. In Section 3, a fuzzy least squares estimation is proposed to estimate parameter $\alpha$. The behaviors of the present estimator are investigated in Section 4, and the proofs of the theorems are given in Section 5. Finally, in Section 6, we deal with the test of the method through simulation studies.

\section{Preliminaries}

In this section, we introduce some basic definitions regarding fuzzy number and fuzzy time series as well as some basic fuzzy theories.

In 1965, Zadeh [16] first introduced the concept of a fuzzy set for modeling the vagueness type of uncertainty. A fuzzy set $A$ defined on the universe $X$ is characterized by a membership function such that $\mu_{A}(x): x \rightarrow[0,1]$. The support of $A$, say $\sup (A)$, is defined by the set $\left\{x \in X \mid \mu_{A}(x)>0\right\}$. For any $\alpha \in(0,1]$, the crisp set $A_{\alpha}=\left\{x \in X \mid \mu_{A}(x)>\alpha\right\}$ is called the $\alpha$-cut of $A$.

A fuzzy subset $A$ of the set of real numbers $R$ with a membership function $\mu_{A}$ is called a fuzzy number if

(1) $A$ is normal, i.e., there exists $x \in R$ such that $\mu_{A}(x)=1$.

(2) $\mu_{A}$ is upper semi-continuous.

(3) $\sup (A)$ is compact.

(4) $A$ is a convex fuzzy set, i.e., $\mu_{A}(\lambda x+(1-\lambda) y) \geq \min \left(\mu_{A}(x), \mu_{A}(y)\right)$ for all $x, y \in R$ and $\lambda \in[0,1]$.

Of extreme interest to us is the LR-fuzzy number whose membership function is defined as follows:

$$
\mu_{A}(x)=\left\{\begin{array}{ll}
L((m-x) / l) & \text { if } x \leq m, \\
R((x-m) / r) & \text { if } x>m,
\end{array} \quad \text { for } x \in R^{1},\right.
$$


where $L: R^{+} \rightarrow[0,1]$ and $R: R^{+} \rightarrow[0,1]$ are fixed left-continuous and non-increasing functions with $L(0)=R(0)=1$, and $R^{+}$denotes the set of nonnegative real numbers. $L$ and $R$ are called left and right shape functions, $m$ the mode of $A$, and $l, r>0$ are the left/right spread of $A$. We abbreviate an LR-fuzzy number by $A=(m, l, r)_{L R}$ and denote the set of all LR-fuzzy numbers by $\mathcal{F}_{L R}\left(R^{1}\right)$. If $L(x)=R(x)=[1-x]^{+}$, then $A=(m, l, r)_{L R}$ is called a triangular fuzzy number and is denoted by $A=(m, l, r)_{\triangle}$. The set of all triangular fuzzy numbers is denoted by $\mathcal{T}\left(R^{1}\right)$. A linear structure is defined on $\mathcal{T}\left(R^{1}\right)$ by

$$
\begin{aligned}
& \left(m_{1}, l_{1}, r_{1}\right)_{\Delta} \oplus\left(m_{2}, l_{2}, r_{2}\right)_{\Delta}=\left(m_{1}+m_{2}, l_{1}+l_{2}, r_{1}+r_{2}\right)_{\Delta}, \\
& \lambda(m, l, r)_{\Delta}= \begin{cases}(\lambda m, \lambda l, \lambda r)_{\triangle} & \text { if } \lambda>0, \\
(\lambda m,-\lambda r,-\lambda l)_{\triangle} & \text { if } \lambda<0, \\
(0,0,0)_{\triangle} & \text { if } \lambda=0 .\end{cases}
\end{aligned}
$$

Furthermore, Diamond [17] gave a metric $d$ on the space $\mathcal{T}\left(R^{1}\right)$ of all triangular fuzzy numbers by

$$
d^{2}(X, Y)=\left[y-\eta^{l}-\left(x-\xi^{l}\right)\right]^{2}+\left[y+\eta^{r}-\left(x+\xi^{r}\right)\right]^{2}+(y-x)^{2},
$$

where $X=\left(x, \xi^{l}, \xi^{r}\right)_{\triangle}$ and $Y=\left(y, \eta^{l}, \eta^{r}\right)_{\triangle}$ are two triangular fuzzy numbers in $\mathcal{T}\left(R^{1}\right)$.

In this paper, we will use the modified metric

$$
d_{H}^{2}(X, Y)=\left\{\left(\left(y-\eta^{l}\right)+y+\left(y+\eta^{r}\right)\right) / 3-\left(\left(x-\xi^{l}\right)+x+\left(x+\xi^{r}\right)\right) / 3\right\}^{2},
$$

which is defined by Kim et al. [15]. By using this modified metric, they have obtained the asymptotic theory of least squares estimator in a fuzzy linear regression model.

Based upon the fuzzy set theory, fuzzy time series models have been defined and studied by Song and Chissom [1]. Let $Y(t)(t=\ldots, 0,1,2, \ldots)$ be a subset of $R^{1}$ in which the universe of fuzzy sets $f_{i}(t)(i=1,2, \ldots, m)$ is defined and let $\mathbf{F}(\mathbf{t})$ be a collection of $f_{i}(t)(i=1,2, \ldots, m)$. Then $\mathbf{F}(\mathbf{t})$ is called a fuzzy time series on $Y(t)(t=\ldots, 0,1,2, \ldots)$.

It can be seen from the above definition that $\mathbf{F}(\mathbf{t})$ is a collection of $f_{i}(t)(i=1,2, \ldots, m)$ which are fuzzy sets defined on $R^{1}$ for a given $t \in T$. The main difference between the traditional time series and the fuzzy time series is that the former has numerical values as its observations, while the latter has fuzzy sets as its observations. One of the application areas of fuzzy time series is the forecasting problems under a fuzzy environment in which no numerical historical data are available but linguistic ones.

\section{Fuzzy least squares estimators}

In this section, we first present an estimator of parameter. Suppose then that we have observations $\left\{X_{t}, t=0,1, \ldots, T\right\}$ from model (1.1). We are interested in estimating $\alpha$ by trying to minimize the conditional sum of squares

$$
Q(\alpha)=\sum_{t=1}^{T} d_{H}^{2}\left(X_{t}, \alpha X_{t-1}\right) .
$$


Recalling the definition of the linear structure on $\mathcal{T}\left(R^{1}\right)$, we have

$$
\begin{aligned}
& \alpha \geq 0, \quad \alpha X_{t-1}=\left(\alpha x_{t-1}, \alpha \xi_{t-1}^{l}, \alpha \xi_{t-1}^{r}\right)_{\triangle^{\prime}} \\
& \alpha<0, \quad \alpha X_{t-1}=\left(\alpha x_{t-1},-\alpha \xi_{t-1}^{r},-\alpha \xi_{t-1}^{l}\right)_{\triangle} .
\end{aligned}
$$

This implies

$$
\begin{aligned}
Q(\alpha) & =\sum_{t=1}^{T} d_{H}^{2}\left(X_{t}, \alpha X_{t-1}\right) \\
& =\sum_{t=1}^{T}\left[x_{t}+\left(\xi_{t}^{r}-\xi_{t}^{l}\right) / 3-\left(\alpha x_{t-1}+\left(\alpha\left(\xi_{t-1}^{r}-\xi_{t-1}^{l}\right)\right) / 3\right)\right]^{2} .
\end{aligned}
$$

The estimation is actually obtained by solving the following least squares equation:

$$
\begin{aligned}
\frac{\partial Q(\alpha)}{\partial \alpha}= & -2 \sum_{t=1}^{T}\left(\left(x_{t}+\left(\xi_{t}^{r}-\xi_{t}^{l}\right) / 3-\left(\alpha x_{t-1}+\left(\alpha\left(\xi_{t-1}^{r}-\xi_{t-1}^{l}\right)\right) / 3\right)\right)\right. \\
& \left.\times\left(x_{t-1}+\left(\xi_{t-1}^{r}-\xi_{t-1}^{l}\right) / 3\right)\right) \\
= & 0 .
\end{aligned}
$$

The solution of equation (3.2) is termed the fuzzy least squares estimation (FLSE) of $\alpha$ and denoted by $\hat{\alpha}$.

Let

$$
W_{t}=x_{t}+\left(\xi_{t}^{r}-\xi_{t}^{l}\right) / 3, \quad t=0,1,2, \ldots
$$

Then

$$
\hat{\alpha}=\left(\sum_{t=1}^{T} W_{t} W_{t-1}\right) /\left(\sum_{t=1}^{T} W_{t-1}^{2}\right) .
$$

Note that if observations $\left\{X_{t}, t=0,1, \ldots, T\right\}$ of model (1.1) are crisp, i.e., $\xi_{t}^{r}=\xi_{t}^{l}=0, \theta_{t}^{l}=$ $\theta_{t}^{r}=0$, then equation (3.2) and estimator (3.3) coincide with the classical least squares method.

\section{Asymptotic normality and forecasting}

In this section, we discuss the properties of the estimator. In order to obtain these properties, we need the following assumptions:

(A1) $\left\{\left(\varepsilon_{t}, \theta_{t}^{l}, \theta_{t}^{r}\right): t=1,2, \ldots\right\}$ is a sequence of independent and identically distributed random vectors.

(A2) $E\left[\varepsilon_{t}\right]=0, E\left[\theta_{t}^{l}\right]=E\left[\theta_{t}^{r}\right]$.

(A3) $\operatorname{Var}\left[\varepsilon_{t}\right]+\operatorname{Var}\left[\theta_{t}^{r}\right]+\operatorname{Var}\left[\theta_{t}^{l}\right]<\infty$.

The following two lemmas are given by Diananda [18].

Lemma 4.1 Let $y_{1}, y_{2}, \ldots$, be a sequence of random variables such that the distribution of $\left(y_{t+t_{1}}, y_{t+t_{2}}, \ldots, y_{t+t_{n}}\right)$ is independent of $t$ for every $0 \leq t_{1}<t_{2}<\cdots<t_{n}$ and $n$ and such that this collection is independent of $\left(y_{s+s_{1}}, y_{s+s_{2}}, \ldots, y_{s+s_{p}}\right)$ for every $0 \leq s_{1}<s_{2}<\cdots<s_{p}$ and 
$p$ if $s>t+t_{n}+m$. Assume Ey $y_{t}=0, E y_{t}^{2}<\infty$, where $p$ and $m$ are positive integers. Then $\sum_{t=1}^{T} y_{t} / \sqrt{T}$ has a limiting normal distribution with mean 0 and variance $E y_{1}^{2}+2 E y_{1} y_{2}+$ $\cdots+2 E y_{1} y_{m+1}$.

Lemma 4.2 Suppose that $\left\{H_{T}\right\},\left\{Z_{T, S}\right\},\left\{X_{T, S}\right\}$ are sequences of random variables. Let $H_{T}=Z_{T, S}+X_{T, S}, T=1,2, \ldots, S=1,2, \ldots$ such that $E X_{T, S}^{2} \leq M_{S}, \lim _{S \rightarrow \infty} M_{S}=0, P\left\{Z_{T, S} \leq\right.$ $z\}=F_{T, S}(z) \rightarrow F_{S}(z)$, as $T \rightarrow \infty, \lim _{S \rightarrow \infty} F_{S}(z)=F(z)$ at every continuity point. Then $\lim _{T \rightarrow \infty} P\left\{H_{T} \leq z\right\}=F(z)$ at every continuity point of $F(z)$.

Now, we state our main results in the following theorems.

Theorem 4.1 Under the conditions of (A1)-(A3), let $M_{T}=\sum_{t=1}^{T} W_{t} W_{t-1}-\alpha \sum_{t=1}^{T} W_{t-1}^{2}$. Then $M_{T} / \sqrt{T} \stackrel{L}{\rightarrow} N\left(0, \sigma^{4} /\left(1-\alpha^{2}\right)\right)$, as $T \rightarrow \infty$, where $\sigma^{2}=\operatorname{Var}\left[\varepsilon_{t}+\left(\theta_{t}^{r}-\theta_{t}^{l}\right) / 3\right]$, the notation $\stackrel{L}{\rightarrow}$ stands for convergence in distribution.

Theorem 4.2 Under the conditions of (A1)-(A3), let $B_{T}=\sum_{t=1}^{T} W_{t-1}^{2}$. Then $B_{T} / T \stackrel{p}{\rightarrow}$ $\sigma^{2} /\left(1-\alpha^{2}\right)$, as $T \rightarrow \infty$, where $\sigma^{2}=\operatorname{Var}\left[\varepsilon_{t}+\left(\theta_{t}^{r}-\theta_{t}^{l}\right) / 3\right]$, where the notation $\stackrel{p}{\rightarrow}$ stands for convergence in probability.

Theorem 4.3 Under the conditions of (A1)-(A3), we have $\sqrt{T}(\hat{\alpha}-\alpha) \stackrel{L}{\rightarrow} N\left(0,1-\alpha^{2}\right)$ as $T \rightarrow \infty$.

Suppose that we have observations $\left\{X_{t}, t=0,1, \ldots, T\right\}$ from model (1.1). Based on the estimator of $\hat{\alpha}$, we can obtain the following forecasting procedure:

1. One-step forecasting: $\hat{X}_{T+1}=\hat{\alpha} X_{T}$;

2. Two-step forecasting: $\hat{X}_{T+2}=\hat{\alpha} X_{T+1}$;

3. $n$-step forecasting: $\hat{X}_{T+n}=\hat{\alpha} X_{T+n-1}$.

\section{Proofs of the theorems}

In this section, we give the proofs of the theorems.

Proof of Theorem 4.1 By (3.1) we have

$$
\begin{aligned}
& \alpha \geq 0, \quad X_{t}=\alpha X_{t-1} \oplus \Phi_{t}=\left(\alpha x_{t-1}+\varepsilon_{t}, \alpha \xi_{t-1}^{l}+\theta_{t}^{l}, \alpha \xi_{t-1}^{r}+\theta_{t}^{r}\right)_{\triangle} \\
& \alpha<0, \quad X_{t}=\alpha X_{t-1} \oplus \Phi_{t}=\left(\alpha x_{t-1}+\varepsilon_{t},-\alpha \xi_{t-1}^{r}+\theta_{t}^{l},-\alpha \xi_{t-1}^{l}+\theta_{t}^{r}\right)_{\triangle} .
\end{aligned}
$$

Note that for all $|\alpha|<1$,

$$
\begin{aligned}
& x_{t}=\alpha x_{t-1}+\varepsilon_{t}, \\
& \left(\xi_{t}^{r}-\xi_{t}^{l}\right) / 3=\left[\alpha\left(\xi_{t-1}^{r}-\xi_{t-1}^{l}\right)+\left(\theta_{t}^{r}-\theta_{t}^{l}\right)\right] / 3 .
\end{aligned}
$$

Let $u_{t}=\varepsilon_{t}+\left(\theta_{t}^{r}-\theta_{t}^{l}\right) / 3$, then

$$
\begin{aligned}
W_{t} & =x_{t}+\left(\xi_{t}^{r}-\xi_{t}^{l}\right) / 3 \\
& =\alpha x_{t-1}+\varepsilon_{t}+\left[\alpha\left(\xi_{t-1}^{r}-\xi_{t-1}^{l}\right)+\left(\theta_{t}^{r}-\theta_{t}^{l}\right)\right] / 3 \\
& =\alpha W_{t-1}+u_{t} .
\end{aligned}
$$


This means that

$$
\begin{aligned}
M_{T} & =\sum_{t=1}^{T} W_{t} W_{t-1}-\alpha \sum_{t=1}^{T} W_{t-1}^{2} \\
& =\sum_{t=2}^{T} u_{t} u_{t-1}+\alpha \sum_{t=3}^{T} u_{t} u_{t-2}+\cdots+\alpha^{T-2} u_{T} u_{1}+W_{0} \sum_{t=1}^{T} \alpha^{t-1} u_{t} .
\end{aligned}
$$

Because the last term has mean 0 and variance $W_{0}^{2} \sigma^{2}\left(1-\alpha^{2 T}\right) /\left(1-\alpha^{2}\right)$, using Chebyshev's inequality, we have

$$
\left(W_{0} \sum_{t=1}^{T} \alpha^{t-1} u_{t}\right) / \sqrt{T} \stackrel{p}{\rightarrow} 0, \quad \text { as } T \rightarrow \infty
$$

Let

$$
\begin{aligned}
& H_{T}=\left(\sum_{t=2}^{T} u_{t} u_{t-1}+\alpha \sum_{t=3}^{T} u_{t} u_{t-2}+\cdots+\alpha^{T-2} u_{T} u_{1}\right) / \sqrt{T}, \\
& Z_{T, S}= \begin{cases}\frac{\sum_{t=2}^{T} u_{t} u_{t-1}+\alpha \sum_{t=3}^{T} u_{t} u_{t-2}+\cdots+\alpha^{S} \sum_{t=S+2}^{T} u_{t} u_{t-S-1}}{\sqrt{T}}, & S \leq T-2, \\
H_{T}, & S>T-2,\end{cases} \\
& X_{T, S}=H_{T}-Z_{T, S} .
\end{aligned}
$$

Since $H_{T}$ is a linear combination of terms $u_{t} u_{s}(t \neq s)$, and each term is uncorrelated with other terms, we have

$$
\begin{aligned}
& E\left[X_{T, S}^{2}\right]<\sigma^{4} \alpha^{2(S+1)} /\left(1-\alpha^{2}\right), \\
& \lim _{S \rightarrow \infty} \sigma^{4} \alpha^{2(S+1)} /\left(1-\alpha^{2}\right)=0 .
\end{aligned}
$$

In what follows, we prove that

$$
Z_{T, S} \stackrel{L}{\rightarrow} N\left(0, \sigma^{4}\left(1-\alpha^{2(S+1)}\right) /\left(1-\alpha^{2}\right)\right), \quad \text { as } T \rightarrow \infty .
$$

Further, let

$$
Z_{T, S}^{*}=\left(\sum_{t=S+2}^{T}\left[u_{t} u_{t-1}+\alpha u_{t} u_{t-2}+\cdots+\alpha^{S} u_{t} u_{t-S-1}\right]\right) / \sqrt{T}
$$

Note that $Z_{T, S}^{*}$ and $Z_{T, S}$ have the same limiting distribution as $T \rightarrow \infty$. Next, we show that

$$
Z_{T, S}^{*} \stackrel{L}{\rightarrow} N\left(0, \sigma^{4}\left(1-\alpha^{2(S+1)}\right) /\left(1-\alpha^{2}\right)\right), \quad \text { as } T \rightarrow \infty .
$$

Now, let

$$
y_{t}=u_{t} u_{t-1}+\alpha u_{t} u_{t-2}+\cdots+\alpha^{S} u_{t} u_{t-S-1} .
$$


Then

$$
\begin{aligned}
& E y_{t}^{2}=\sigma^{4}\left(1-\alpha^{2(S+1)}\right) /\left(1-\alpha^{2}\right), \\
& E\left(y_{t} y_{l}\right)=0 \quad(t \neq l) .
\end{aligned}
$$

Thus, combining Lemma 4.1, we prove (5.5). The theorem follows from (5.1)-(5.5) and Lemma 4.2.

Proof of Theorem 4.2 Note that

$$
\begin{aligned}
B_{T}= & \sum_{t=1}^{T} W_{t-1}^{2} \\
= & W_{0}^{2}+\left(u_{1}+\alpha W_{0}\right)^{2}+\cdots+\left(u_{T-1}+\alpha u_{T-2}+\cdots+\alpha^{T-1} W_{0}\right)^{2} \\
= & {\left[u_{1}^{2}\left(1+\alpha^{2}+\cdots+\alpha^{2(T-2)}\right)+\cdots+u_{T-1}^{2}\right] } \\
& +2\left[\alpha\left(u_{2} u_{1}+\cdots+u_{T-1} u_{T-2}\right)+\cdots+\alpha^{T-2} u_{T-1} u_{1}\right] \\
& +2 W_{0}\left[u_{1}\left(\alpha+\alpha^{3}+\cdots+\alpha^{2 T-3}\right)+\cdots+\alpha^{T-1} u_{T-1}\right] \\
& +W_{0}^{2}\left[1+\alpha^{2}+\cdots+\alpha^{2(T-1)}\right] \\
= & B_{T_{1}}+B_{T_{2}}+B_{T_{3}}+B_{T_{4}} .
\end{aligned}
$$

It is easy to see that

$$
B_{T_{4}} / T \rightarrow 0, \quad \text { as } T \rightarrow \infty
$$

Because $E\left(B_{T_{3}} / T\right)=0$ and $E\left(B_{T_{3}} / T\right)^{2} \leq 4 W_{0}^{2} \sigma^{2} \alpha^{2} /\left(T\left(1-\alpha^{2}\right)^{2}\right)$, we have

$$
B_{T_{3}} / T \stackrel{p}{\rightarrow} 0, \quad \text { as } T \rightarrow \infty .
$$

Further, from

$$
\begin{aligned}
E\left(B_{T_{2}} / T\right) & =0, \\
E\left(B_{T_{2}} / T\right)^{2} & =4\left[\sigma^{4}\left(\alpha^{2}(T-2)+\alpha^{4}(T-3)+\cdots+\alpha^{2(T-2)}\right)\right] / T^{2} \\
& <4\left[\sigma^{4}\left(1+\alpha^{2}+\cdots\right)\right] / T \rightarrow 0, \quad \text { as } T \rightarrow \infty,
\end{aligned}
$$

similarly, we get

$$
B_{T_{2}} / T \stackrel{p}{\rightarrow} 0, \quad \text { as } T \rightarrow \infty .
$$

Lastly, we will show that $B_{T_{1}} / T \stackrel{p}{\rightarrow} \sigma^{2} /\left(1-\alpha^{2}\right)$. Observe that

$$
\begin{aligned}
\frac{1}{1-\alpha^{2}} \sum_{t=1}^{T-1} u_{t}^{2}-B_{T_{1}} & =u_{1}^{2}\left(\alpha^{2(T-1)}+\alpha^{2 T}+\cdots\right)+\cdots+u_{T-1}^{2}\left(\alpha^{2}+\alpha^{4}+\cdots\right) \\
& =u_{1}^{2} \alpha^{2(T-1)} /\left(1-\alpha^{2}\right)+\cdots+\alpha^{2} u_{T-1}^{2} /\left(1-\alpha^{2}\right) .
\end{aligned}
$$


Since this is a nonnegative random variable with expected value

$$
\left(\alpha^{2} \sigma^{2}\left(1-\alpha^{2(T-1)}\right)\right) /\left(1-\alpha^{2}\right)^{2}
$$

using Markov's inequality, we have

$$
\left(\frac{1}{1-\alpha^{2}} \sum_{t=1}^{T-1} u_{t}^{2}-B_{T_{1}}\right) / T \stackrel{p}{\rightarrow} 0, \quad \text { as } T \rightarrow \infty
$$

Further, by the law of large numbers, we get

$$
\left(\frac{1}{1-\alpha^{2}} \sum_{t=1}^{T-1} u_{t}^{2}\right) / T \stackrel{p}{\rightarrow} \sigma^{2} /\left(1-\alpha^{2}\right), \quad \text { as } T \rightarrow \infty .
$$

Then it holds that

$$
B_{T_{1}} / T \stackrel{p}{\rightarrow} \sigma^{2} /\left(1-\alpha^{2}\right) \quad \text { as } T \rightarrow \infty
$$

This, together with (5.6)-(5.9), completes the proof.

Proof of Theorem 4.3 Note that

$$
\sqrt{T}(\hat{\alpha}-\alpha)=\left(M_{T} / \sqrt{T}\right) /\left(B_{T} / T\right)
$$

With the application of Slusky's theorem and Theorems 4.1 and 4.2, we prove Theorem 4.3.

\section{Simulation results}

In this section, we conduct some simulations to show the finite performance of the proposed method. The simulation uses the fuzzy autoregressive model (1.1).

In the first simulation, we evaluate the finite sample performance of $\hat{\alpha}$. The modes and spreads of fuzzy random errors $\Phi_{t}$ are chosen as random samples from normal and uniform distributions, $N(0,1), U[0,0.5]$, respectively. To assess the sensitivity of the estimates to sample size and parameter, simulations are conducted for different parameter values and sample sizes. In different simulations, we set $\alpha$ equal to $-0.8,-0.6,-0.4,-0.2,0.2$, $0.4,0.6$, and 0.8, respectively. Moreover, the sample sizes used are 50, 100, 300, and 500 . For a particular sample size, 1,000 different sets of data were generated. For each data set, we estimate the parameter $\alpha$ by the proposed estimator in (3.3) and report the average estimates and average mean squared error (MMSE) over 1000 simulations. The results are presented in Table 1.

From Table 1, we see that the estimation procedure works very well. For different sample sizes and different parameters, the average mean squared errors of $\hat{\alpha}$ are very small, and the average mean squared error of $\hat{\alpha}$ decreases as sample size increases. Furthermore, we also find that for the same sample size, the average mean squared error of $\hat{\alpha}$ decreases as the absolute value of the parameter becomes large. 
Table 1 Average estimates and average mean squared errors

\begin{tabular}{rllllllll}
\hline $\boldsymbol{\alpha}$ & $\boldsymbol{n}=\mathbf{5 0}$ & & $\boldsymbol{n}=\mathbf{1 0 0}$ & & $\boldsymbol{n}=\mathbf{3 0 0}$ & \multicolumn{3}{c}{$\mathbf{n = 5 0 0}$} \\
& FLSE & MMSE & FLSE & MMSE & FLSE & MMSE & FLSE & MMSE \\
\hline-0.8 & -0.7723 & 0.0096 & -0.7829 & 0.0045 & -0.7961 & 0.0012 & -0.7962 & $7.4056 \times 10^{-4}$ \\
-0.6 & -0.5834 & 0.0133 & -0.5892 & 0.0066 & -0.5979 & 0.0021 & -0.5986 & 0.0013 \\
-0.4 & -0.3844 & 0.0168 & -0.3916 & 0.0080 & -0.3960 & 0.0028 & -0.3985 & 0.0017 \\
-0.2 & -0.1935 & 0.0180 & -0.1955 & 0.0099 & -0.1981 & 0.0034 & -0.1989 & 0.0019 \\
0.2 & 0.1899 & 0.0182 & 0.1932 & 0.0095 & 0.1992 & 0.0030 & 0.1999 & 0.0017 \\
0.4 & 0.3844 & 0.0169 & 0.3913 & 0.0079 & 0.3980 & 0.0028 & 0.3990 & 0.0017 \\
0.6 & 0.5741 & 0.0130 & 0.5916 & 0.0067 & 0.5971 & 0.0020 & 0.5975 & 0.0012 \\
0.8 & 0.7693 & 0.0097 & 0.7858 & 0.0042 & 0.7941 & 0.0013 & 0.7978 & $7.3562 \times 10^{-4}$ \\
\hline
\end{tabular}

Table 2 Square sum of the forecast error

\begin{tabular}{|c|c|c|c|}
\hline$\alpha$ & SSFE & $\alpha$ & SSFE \\
\hline 0.1 & 24.4591 (1.8413) & 0.2 & $45.4120(27.6935)$ \\
\hline 0.3 & $30.9102(23.8170)$ & 0.4 & 3.1675 (1.0148) \\
\hline 0.5 & $17.8231(15.4548)$ & 0.6 & $10.5958(7.0525)$ \\
\hline 0.7 & $5.5013(3.7557)$ & 0.8 & $6.6267(3.0133)$ \\
\hline 0.9 & 18.9895 (15.8378) & 0.98 & 22.7701 (9.1734) \\
\hline
\end{tabular}

In the second simulation, we illustrate the performance of the forecast procedure proposed above. We compare model (1.1) with the ordinary autoregressive model $X_{t}=\alpha X_{t-1}+$ $\varepsilon_{t}$, where $\varepsilon_{t}$ is error sequence.

Firstly, we produce 50 samples $X_{1}, X_{2}, \ldots, X_{50}$ of model (1.1). Then by using the first 30 samples $X_{1}, X_{2}, \ldots, X_{30}$, we can obtain the estimator $\hat{\alpha}$. We forecast $X_{31}, X_{2}, \ldots, X_{50}$. The modes and spreads of the fuzzy random errors $\Phi_{t}$ are chosen as random samples from normal and uniform distributions, $N(0,4), U[0,5]$, respectively. For the ordinary autoregressive model, we only forecast modal values based on the above fuzzy data. We compare the square sum of the forecast error (SSFE) of modal values. The results are presented in Table 2, and the figures in parentheses are those for model (1.1). From Table 2, we can see that model (1.1) has less forecast error than the ordinary autoregressive model.

\section{Conclusions}

In this paper, we introduce a new fuzzy autoregressive model. The model can be considered as an extension of the standard autoregressive model since crisp values can be treated as degenerated fuzzy numbers. Least squares estimation is derived, and the asymptotic distribution of the proposed estimator is established. This estimation procedure is well defined because if we use crisp data instead of fuzzy observations, then our estimation reduces to the classical estimation. The simulation results indicate that the least squares estimation performs very well.

It should be noted that we here discuss the first-order autoregressive model with triangular fuzzy data. Further research needs to be undertaken to discover the analogous results for other models, such as the unstable first-order autoregressive model or high-order autoregressive model, with more complicated metrics and/or other types of fuzzy data. It is also interesting to consider the problem of testing hypotheses about the parameters in these models. 
Authors' contributions

The first author, who is a PhD, and the second author jointly worked on deriving the results. Both authors read and approved the final manuscript.

\section{Author details}

${ }^{1}$ College of Mathematics, Jilin Normal University, Siping, 136000, P.R. China. ${ }^{2}$ Public Foreign Languages Department, Jilin Normal University, Siping, 136000, P.R. China.

\section{Acknowledgements}

This work is supported by the Science and Technology Development Program of Jilin Province (201201082).

Received: 27 October 2012 Accepted: 1 February 2013 Published: 18 February 2013

\section{References}

1. Song, Q, Chissom, BS: Fuzzy time series and its models. Fuzzy Sets Syst. 54, 269-277 (1993)

2. Song, Q, Chissom, BS: Forecasting enrollments with fuzzy time series - part I. Fuzzy Sets Syst. 54, 1-9 (1993)

3. Song, Q, Chissom, BS: Forecasting enrollments with fuzzy time series - part II. Fuzzy Sets Syst. 62, 1-8 (1994)

4. Chen, SM: Forecasting enrollments based on fuzzy time series. Fuzzy Sets Syst. 81, 311-319 (1996)

5. Hwang, JR, Chen, SM, Lee, CH: Handling forecasting problems using fuzzy time series. Fuzzy Sets Syst. 100, 217-228 (1998)

6. Sullivan, J, Woodall, WH: A comparison of fuzzy forecasting and Markov modeling. Fuzzy Sets Syst. 64, 279-293 (1994)

7. Chen, SM, Hwang, JR: Temperature prediction using fuzzy time series. IEEE Trans. Syst. Man Cybern., Part B, Cybern. 30, 263-275 (2000)

8. Huarng, K: Heuristic models of fuzzy time series for forecasting. Fuzzy Sets Syst. 123, 369-386 (2001)

9. Huarng, K, Yu, HK: A Type 2 fuzzy time series model for stock index forecasting. Phys. A, Stat. Mech. Appl. 353, 445-462 (2005)

10. Huarng, K, Yu, HK: The application of neural networks to forecast fuzzy time series. Phys. A, Stat. Mech. Appl. 363, 481-491 (2006)

11. Yu, HK: A refined fuzzy time-series model for forecasting. Phys. A, Stat. Mech. Appl. 346, 657-681 (2005)

12. Yu, HK: Weighted fuzzy time series models for TAIEX forecasting. Phys. A, Stat. Mech. Appl. 349, 609-624 (2005)

13. Ozawa, K, Watanabe, T, Kanke, M: Fuzzy autoregressive model and its applications. In: 1997 First International Conference on Knowledge-Based Intelligent Electronic Systems, pp. 112-117 (1997)

14. Niimura, T, Ko, HS, Ozawa, K: A day-ahead electricity price prediction based on a fuzzy-neuro autoregressive model in a deregulated electricity market. In: Proceedings of the 2002 International Joint Conference on Neural Networks, vol. 2, pp. 1362-1366 (2002)

15. Kim, HK, Yoon, JH, Li, Y: Asymptotic properties of least squares estimation with fuzzy observations. Inf. Sci. 178, 439-451 (2008)

16. Zadeh, LA, Klir, GJ, Yuan, B: Fuzzy Sets, Fuzzy Logic, and Fuzzy Systems: Selected Papers. World Scientific, Singapore (1996)

17. Diamond, P: Fuzzy least squares. Inf. Sci. 46, 141-157 (1998)

18. Diananda, PH: Some probability limit theorems with statistical applications. Proc. Camb. Philos. Soc. 49, $239-246$ (1953)

doi:10.1186/1029-242X-2013-56

Cite this article as: Zhao and Peng: Asymptotic properties of least squares estimation for a new fuzzy autoregressive model. Journal of Inequalities and Applications 2013 2013:56.

\section{Submit your manuscript to a SpringerOpen ${ }^{\circ}$ journal and benefit from:}

- Convenient online submission

- Rigorous peer review

Immediate publication on acceptance

- Open access: articles freely available online

- High visibility within the field

- Retaining the copyright to your article 